\title{
QUASICONTINUOUS FUNCTIONS, DENSELY CONTINUOUS FORMS AND COMPACTNESS
}

\author{
L'ubica Holá - Dušan HolÝ
}

\begin{abstract}
Let $X$ be a locally compact space. A subfamily $\mathcal{F}$ of the space $D^{\star}(X, \mathbb{R})$ of densely continuous forms with nonempty compact values from $X$ to $\mathbb{R}$ equipped with the topology $\tau_{U C}$ of uniform convergence on compact sets is compact if and only if $\{\sup (F): F \in \mathcal{F}\}$ is compact in the space $Q(X, \mathbb{R})$ of quasicontinuous functions from $X$ to $\mathbb{R}$ equipped with the topology $\tau_{U C}$.
\end{abstract}

\section{Introduction}

Quasicontinuous functions were introduced by Kempisty in 1932 in [14]. They are important in many areas of mathematics. They found applications in the study of minimal USCO and minimal CUSCO maps [7], 8], in the study of topological groups [3], [16], [18, in proofs of some generalizations of Michael's selection theorem [5], in the study of extensions of densely defined continuous functions [6], in the study of dynamical systems [4]. The quasicontinuity is also used in the study of CHART groups [17.

Densely continuous forms were introduced by $\mathrm{Hammer}$ and $\mathrm{McCoy}$ in [12]. Densely continuous forms can be considered as set-valued mappings from a topological space $X$ into a topological space $Y$ which have a kind of minimality property found in the theory of minimal USCO mappings. In particular, every minimal USCO mapping from a Baire space into a metric space is a densely continuous form. There is also a connection between differentiability properties of convex functions and densely continuous forms as expressed via the subdifferentials of convex functions, which are a kind of convexification of minimal USCO mappings [12].

(C) 2017 Mathematical Institute, Slovak Academy of Sciences. 2010 Mathematics Subject Classification: 54C 35, 54B20, 54C08.

Keywords: quasicontinuous function, densely continuous form, compactness, densely equiquasicontinuous, pointwise bounded, boundedly compact metric space.

Supported by the grant Vega 2/0006/16. 


\section{L'UBICA HOLÁ - DUŠAN HOLÝ}

In 12 the authors proved the Ascoli-type theorem for densely continuous forms from a locally compact space to a boundedly compact metric space. In our paper we present new characterizations of compact subsets of $\left(D^{\star}(X, \mathbb{R}), \tau_{U C}\right)$ via quasicontinuous selections of elements of $D^{\star}(X, \mathbb{R})$.

\section{Preliminaries}

In what follows let $X, Y$ be Hausdorff topological spaces, $\mathbb{Z}^{+}$be the set of positive integers, $\mathbb{R}$ be the space of real numbers with the usual metric. The symbol $\bar{A}$ will stand for the closure of the set $A$ in a topological space.

A function $f: X \rightarrow Y$ is quasicontinuous [19] at $x \in X$ if for every open set $V \subset Y, f(x) \in V$ and every open set $U \subset X, x \in U$ there is a nonempty open set $W \subset U$ such that $f(W) \subset V$. If $f$ is quasicontinuous at every point of $X$, we say that $f$ is quasicontinuous.

Denote by $F(X, Y)$ the set of all functions from $X$ to $Y$ and by $Q(X, Y)$ the set of all quasicontinuous functions in $F(X, Y)$.

By $2^{Y}$ we denote the space of all closed subsets of $Y$ and by $C L(Y)$ the space of all nonempty closed subsets of $Y$. By $K(Y)$ we denote the space of all nonempty compact subsets of $Y$. The space of all functions from $X$ to $2^{Y}$ we denote by $F\left(X, 2^{Y}\right)$. We also call the functions from $F\left(X, 2^{Y}\right)$ set-valued functions, or multifunctions, from $X$ to $Y$. In our paper, we will identify functions and setvalued functions with their graphs.

To define a densely continuous form from $X$ to $Y[12$, denote by $D C(X, Y)$ the set of all functions $f \in F(X, Y)$ such that the set $C(f)$ of points of continuity of $f$ is dense in $X$. We call such functions densely continuous.

Of course, $D C(X, Y)$ contains the set $C(X, Y)$ of all continuous functions from $X$ to $Y$. If $Y=\mathbb{R}$ and $X$ is a Baire space, then all upper and lower semicontinuous functions on $X$ belongs to $D C(X, Y)$ and if $X$ is a Baire space and $Y$ is a metric space, then every quasicontinuous function $f: X \rightarrow Y$ has a dense $G_{\delta^{-}}$set $C(f)$ of the points of continuity of $f[19]$; i.e., $Q(X, Y) \subset D C(X, Y)$. Notice that points of continuity and quasicontinuity of functions are studied in [2].

For every $f \in D C(X, Y)$, we denote by $\overline{f \uparrow C(f)}$ the closure of the graph

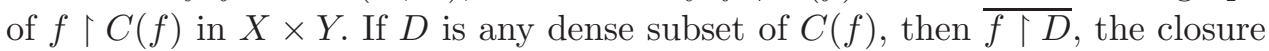
of the graph of $f \uparrow D$ in $X \times Y$ is equal to $\overline{f \uparrow C(f)}$. We define the set $D(X, Y)$ of densely continuous forms by

$$
D(X, Y)=\{\overline{f \uparrow C(f)}: f \in D C(X, Y)\} .
$$

Densely continuous forms from $X$ to $Y$ may be considered as set-valued mappings, where for each $x \in X$ and $F \in D(X, Y), F(x)=\{y \in Y:(x, y) \in F\}$. 
Definition 2.1 (19]). Let $X, Y$ be topological spaces and $F$ be a set-valued mapping from $X$ to $Y$. $F$ is lower quasicontinuous at a point $x \in X$ if for every open set $G$ intersecting $F(x)$ and every open set $H$ containing $x$ there is a nonempty open set $U \subset H$ such that $G \cap F(u) \neq \emptyset$ for any $u \in U$. F is lower quasicontinuous if it is lower quasicontinuous at any point of $X$.

We will study relations between quasicontinuous functions and densely continuous forms.

The following two propositions are clear.

Proposition 2.2. Let $X, Y$ be topological spaces and $F$ be a densely continuous form from $X$ to $Y$. Then $F$ is lower quasicontinuous.

Proposition 2.3. Let $X, Y$ be topological spaces. Let $f$ be a function from $X$ to $Y$. If $f \subset \overline{f\lceil C(f)}$, then $f$ is a quasicontinuous function and $f \in D C(X, Y)$.

Remark 2.4. It is easy to see that if $f \in Q(X, Y)$ and $D$ is a dense subset of $X$, then $\bar{f}=\overline{f \uparrow D}$.

Denote by

$$
D^{\bullet}(X, Y)=\{F \in D(X, Y): F(x) \neq \emptyset \text { for every } x \in X\} .
$$

We have the following characterizations of the elements of $D^{\bullet}(X, Y)$ [7]. For a reader's convenience we will prove it.

Theorem 2.5. Let $X$ be a Baire space and $Y$ be a metric space. Let $F$ be a set-valued mapping from $X$ to $Y$. The following are equivalent:

(1) $F \in D^{\bullet}(X, Y)$.

(2) There is a quasicontinuous function $f: X \rightarrow Y$ such that $\bar{f}=F$.

(3) Every selection $f$ of $F$ is quasicontinuous and $\bar{f}=F$.

Pr o of. $(1) \Rightarrow(3)$ Let $f$ be a selection of $F$. There is $g \in D C(X, Y)$ such that $F=\overline{g\lceil C(g)}$. Of course $F(x)=\{g(x)\}$ for every $x \in C(g)$; i.e., $f(x)=g(x)$ for every $x \in C(g)$. It is easy to verify that $C(g) \subset C(f)$. (Let $x \in C(g)$. Suppose $x \notin C(f)$. There is an open neighbourhood $V$ of $f(x)$ such that for every open neighbourhood $U$ of $x$ there is $x_{U} \in U$ with $f\left(x_{U}\right) \notin V$. Let $H$ be an open neighbourhood of $f(x)$ such that $\bar{H} \subset V$. The continuity of $g$ at $x$ implies that there is an open neighbourhood $O$ of $x$ such that $g(O) \subset H$. Then $O \times(Y \backslash \bar{H})$ is a neighbourhood of $\left(x_{O}, f\left(x_{O}\right)\right)$ which has an empty intersection with the graph of $g$, a contradiction, since $f \subset \overline{g \uparrow C(g)}$. )

Since $f \subset F=\overline{g\lceil C(g)} \subset \overline{f \uparrow C(f)}$, by Proposition 2.3 we have that $f$ is quasicontinuous. Of course $F=\bar{f}$.

$(3) \Rightarrow(2)$ is trivial. (2) $\Rightarrow(1)$ is also trivial since if $f \in Q(X, Y)$, then $C(f)$ is a dense $G_{\delta}$-set by [19] and by Remark 2.4 $\bar{f}=\overline{f \uparrow C(f)}=F$. 


\section{L'UBICA HOLÁ - DUŠAN HOLÝ}

Notice that closures of graphs of quasicontinuous functions were studied also in 15 .

Let $(Y, d)$ be a metric space. The open $d$-ball with center $z_{0} \in Y$ and radius $\epsilon>0$ will be denoted by $S_{\epsilon}\left(z_{0}\right)$ and the $\epsilon$-enlargement $\bigcup_{a \in A} S_{\epsilon}(a)$ for a subset $A$ of $Y$ will be denoted by $S_{\epsilon}(A)$.

If $A \in C L(Y)$, the distance functional $d(., A): Y \mapsto[0, \infty)$ is described by the familiar formula

$$
d(z, A)=\inf \{d(z, a): a \in A\} .
$$

Let $A$ and $B$ be nonempty subsets of $(Y, d)$. The excess of $A$ over $B$ with respect to $d$ is defined by the formula

$$
e_{d}(A, B)=\sup \{d(a, B): a \in A\} .
$$

The Hausdorff (extended-valued) metric $H_{d}$ on $C L(Y)$ [1] is defined by

$$
H_{d}(A, B)=\max \left\{e_{d}(A, B), e_{d}(B, A)\right\} .
$$

We will often use the following equality on $C L(Y)$ :

$$
H_{d}(A, B)=\inf \left\{\varepsilon>0: A \subset S_{\varepsilon}(B) \text { and } B \subset S_{\varepsilon}(A)\right\} .
$$

If $(Y, d)$ is a complete metric space, then both $\left(C L(Y), H_{d}\right)$ and $\left(K(Y), H_{d}\right)$ are complete [1].

The topology generated by $H_{d}$ is called the Hausdorff metric topology.

Following [12 we will define the topology $\tau_{p}$ of pointwise convergence on $F\left(X, 2^{Y}\right)$. The topology $\tau_{p}$ of pointwise convergence on $F\left(X, 2^{Y}\right)$ is induced by the uniformity $\mathfrak{U}_{p}$ of pointwise convergence which has a base consisting of sets of the form

$$
W(A, \varepsilon)=\left\{(\Phi, \Psi): \forall x \in A \quad H_{d}(\Phi(x), \Psi(x))<\varepsilon\right\},
$$

where $A$ is a finite set in $X$ and $\varepsilon>0$.

We will define the topology $\tau_{U C}$ of uniform convergence on compact sets on $F\left(X, 2^{Y}\right)\left[12\right.$. This topology is induced by the uniformity $\mathfrak{U}_{U C}$ which has a base consisting of sets of the form

$$
W(K, \varepsilon)=\left\{(\Phi, \Psi): \forall x \in K \quad H_{d}(\Phi(x), \Psi(x))<\varepsilon\right\},
$$

where $K \in K(X)$ and $\varepsilon>0$. The general $\tau_{U C}$-basic neighborhood of $\Phi \in F\left(X, 2^{Y}\right)$ will be denoted by $W(\Phi, K, \varepsilon)$, i.e.,

$$
W(\Phi, K, \varepsilon)=W(K, \varepsilon)[\Phi]=\left\{\Psi: H_{d}(\Phi(x), \Psi(x))<\varepsilon \text { for every } x \in K\right\} .
$$

Finally we will define the topology $\tau_{U}$ of uniform convergence on $F\left(X, 2^{Y}\right)[12$. Let $e$ be the (extended-valued) metric on $F\left(X, 2^{Y}\right)$ defined by

$$
e(\Phi, \Psi)=\sup \left\{H_{d}(\Phi(x), \Psi(x)): x \in X\right\}
$$


for each $\Phi, \Psi \in F\left(X, 2^{Y}\right)$. Then the topology of uniform convergence for the space $F\left(X, 2^{Y}\right)$ is the topology generated by the metric $e$.

We use the symbols $\tau_{p}, \tau_{U C}$ and $\tau_{U}$ also for the topology of pointwise convergence, the topology of uniform convergence on compacta and the topology of uniform convergence on the space of all functions from $X$ to $Y$, respectively.

\section{Densely continuous forms and quasicontinuous functions}

Let $X$ and $Y$ be topological spaces. Define the mapping

$$
\phi: F(X, Y) \rightarrow F\left(X, 2^{Y}\right) \text { as } \phi(f)=\bar{f} .
$$

We say that a metric space $(Y, d)$ is boundedly compact [1 if every closed bounded subset is compact. Therefore $(Y, d)$ is a locally compact, separable metric space and $d$ is complete. In fact, any locally compact, separable metric space has a compatible metric $d$ such that $(Y, d)$ is a boundedly compact space [20].

Proposition 3.1. Let $X$ be a topological space and $(Y, d)$ be a boundedly compact metric space. The mapping $\phi:\left(F(X, Y), \tau_{U}\right) \rightarrow\left(F\left(X, 2^{Y}\right), \tau_{U}\right)$ is continuous.

Proof. Let $\left\{f_{\sigma}: \sigma \in \Sigma\right\}$ be a net in $F(X, Y)$ which uniformly converges to $f \in F(X, Y)$. We show that $\left\{\bar{f}_{\sigma}: \sigma \in \Sigma\right\}$ uniformly converges to $\bar{f}$ in $F\left(X, 2^{Y}\right)$. Suppose that $\left\{\bar{f}_{\sigma}: \sigma \in \Sigma\right\}$ fails to converge uniformly to $\bar{f}$. There exists $\epsilon>0$ such that

$$
\begin{aligned}
& \text { for every } \sigma \in \Sigma \text { there are } \beta_{\sigma} \geq \sigma \text { and } a_{\sigma} \in X \\
& \text { such that } H_{d}\left(\bar{f}\left(a_{\sigma}\right), \bar{f}_{\beta_{\sigma}}\left(a_{\sigma}\right)\right)>\epsilon .
\end{aligned}
$$

There is $\sigma \in \Sigma$ such that $d\left(f_{\eta}(x), f(x)\right)<\epsilon / 4$ for every $\eta \geq \sigma$ and for every $x \in X$. By $(*)$ there are $\beta_{\sigma} \geq \sigma$ and $a_{\sigma} \in X$ such that

$$
H_{d}\left(\bar{f}\left(a_{\sigma}\right), \bar{f}_{\beta_{\sigma}}\left(a_{\sigma}\right)\right)>\epsilon .
$$

So either there exists $s \in \bar{f}\left(a_{\sigma}\right)$ such that $S_{\epsilon}(s) \cap \bar{f}_{\beta_{\sigma}}\left(a_{\sigma}\right)=\emptyset$ or there is $t \in \bar{f}_{\beta_{\sigma}}\left(a_{\sigma}\right)$ such that $S_{\epsilon}(t) \cap \bar{f}\left(a_{\sigma}\right)=\emptyset$.

Suppose there is $s \in \bar{f}\left(a_{\sigma}\right)$ such that $S_{\epsilon}(s) \cap \bar{f}_{\beta_{\sigma}}\left(a_{\sigma}\right)=\emptyset$.

Then there exists a net $\left\{x_{\lambda}: \lambda \in \Lambda\right\}$ converging to $a_{\sigma}$ such that $f_{\beta_{\sigma}}\left(x_{\lambda}\right) \in$ $S_{\epsilon / 2}(s)$ for every $\lambda \in \Lambda$. Since the space $(Y, d)$ is boundedly compact there is a point $u \in \overline{S_{\epsilon / 2}(s)}$ which is a cluster point of the net $\left\{f_{\beta_{\sigma}}\left(x_{\lambda}\right): \lambda \in \Lambda\right\}$ and so $S_{\epsilon}(s) \cap \bar{f}_{\beta_{\sigma}}\left(a_{\sigma}\right) \neq \emptyset$, a contradiction.

The other case is similar.

The following proposition follows from the previous one. 


\section{L'UBICA HOLÁ - DUŠAN HOLÝ}

Proposition 3.2. Let $X$ be a locally compact topological space and $(Y, d)$ be a boundedly compact metric space. Then the mapping $\phi:\left(F(X, Y), \tau_{U C}\right) \rightarrow$ $\left(F\left(X, 2^{Y}\right), \tau_{U C}\right)$ is continuous.

Proposition 3.3. Let $X$ be a locally compact topological space and $(Y, d)$ be a boundedly compact metric space. Let $\mathcal{C}$ be a compact subset of $\left(Q(X, Y), \tau_{U C}\right)$. Then $\mathcal{D}=\{\bar{f}: f \in \mathcal{C}\}$ is a compact subset of $\left(D(X, Y), \tau_{U C}\right)$.

P r o of. The proof follows from Proposition 3.2

The following example shows that Proposition 3.2 does not work for the pointwise topology.

ExAmple 3.4. Let $X=[0,1]$ with the usual topology. Consider the function $f: X \rightarrow \mathbb{R}$ defined by $f(x)=1$ for each $x \in X$ and the functions $f_{n}: X \rightarrow \mathbb{R}$, $n \in \mathbb{Z}^{+}$defined by

$$
f_{n}(x)= \begin{cases}\cos \frac{1}{x}, & x \in\left(0, \frac{1}{2 n \pi}\right] \\ 1, & x \in\{0\} \cup\left(\frac{1}{2 n \pi}, 1\right] .\end{cases}
$$

Then the sequence $\left\{f_{n}: n \in \mathbb{Z}^{+}\right\}$pointwise converges to $f$, but $\left\{\bar{f}_{n}: n \in \mathbb{Z}^{+}\right\}$ does not pointwise converge to $\bar{f}$.

Denote by $D^{\star}(X, \mathbb{R})$ the set of all densely continuous forms with nonempty compact values in $\mathbb{R}$. Define the mapping $\sup : D^{\star}(X, \mathbb{R}) \rightarrow Q(X, \mathbb{R})$ as follows

$$
\sup (F)(x)=\sup F(x) .
$$

We have the following proposition.

Proposition 3.5. Let $X$ be a Baire space. The mapping sup : $D^{\star}(X, \mathbb{R}) \rightarrow$ $Q(X, \mathbb{R})$ is injective.

P r o of. Let $F, G \in D^{\star}(X, \mathbb{R})$ be such that $F \neq G$. By Theorem $2.5 \overline{\sup (F)}=F$ and $\overline{\sup (G)}=G$.

Let $(x, y) \in \overline{\sup (F)} \backslash \overline{\sup (G)}$. Let $U, V$ be open sets in $X$ and $\mathbb{R}$, respectively, such that $x \in U, y \in V$ and $(U \times V) \cap \overline{\sup (G)}=\emptyset$. Let $(z, \sup (F)(z)) \in U \times V$. Thus $\sup F(z) \neq \sup G(z)$.

Remark 3.6. It is easy to see that if $A$ and $B$ are nonempty compact subsets of $\mathbb{R}$, then $d(\sup A, \sup B) \leqq H_{d}(A, B)$.

Theorem 3.7. Let $X$ be a locally compact space. The spaces $\left(D^{\star}(X, \mathbb{R}), \tau_{U C}\right)$ and $\left(\sup \left(D^{\star}(X, \mathbb{R})\right), \tau_{U C}\right)$ are uniformly isomorphic. 
P r o o f. From Remark 3.6 follows that the mapping sup : $\left(D^{\star}(X, \mathbb{R}), \tau_{U C}\right) \rightarrow$ $\left(Q(X, \mathbb{R}), \tau_{U C}\right)$ is uniformly continuous.

To prove that also sup ${ }^{-1}$ is uniformly continuous let $K \in K(X)$ and $\varepsilon>0$. The local compactness of $X$ implies that there is an open set $V$ in $X$ such that $K \subset V$ and $\bar{V}$ is compact. Let $F, G \in D^{\star}(X, \mathbb{R})$ be such that $d(\sup (F)(x)$, $\sup (G)(x))<\varepsilon$ for every $x \in \bar{V}$. We prove that $H_{d}(F, G) \leq \varepsilon$ for every $x \in K$.

By Theorem 2.5 we have $\overline{\sup (F)}=F$ and $\overline{\sup (G)}=G$. Let $x_{0} \in K$ and let $r \in F\left(x_{0}\right)$. Then there is a net $\left\{x_{\sigma}: \sigma \in \Sigma\right\}$ in $V$ such that $\left\{\sup (F)\left(x_{\sigma}\right): \sigma \in \Sigma\right\}$ converges to $r$. The net $\left\{\sup (G)\left(x_{\sigma}\right): \sigma \in \Sigma\right\}$ has a cluster point $s \in G\left(x_{0}\right)$. From this follows that $d\left(r, G\left(x_{0}\right)\right) \leq \varepsilon$. Similarly, we can show that if $s \in G\left(x_{0}\right)$, then $d\left(s, F\left(x_{0}\right)\right) \leq \varepsilon$. Hence $H_{d}\left(F\left(x_{0}\right), G\left(x_{0}\right)\right) \leq \varepsilon$.

The following Lemma will be useful in the proof of the next theorem.

LEMmA 3.8. Let $X$ be a locally compact space and $(Y, d)$ be a metric space. Then $Q(X, Y)$ is a closed subset of $\left(F(X, Y), \tau_{U C}\right)$.

Proof. It is known that the uniform limit of quasicontinuous functions with values in a metric space is quasicontinuous [19].

TheOrem 3.9. Let $X$ be a locally compact topological space. Then the spaces $\left(D^{\star}(X, \mathbb{R}), \mathfrak{U}_{U C}\right)$ and $\left(\sup \left(D^{\star}(X, \mathbb{R})\right), \mathfrak{U}_{U C}\right)$ are complete uniform spaces.

P r o of. Let $\left\{F_{\sigma}: \sigma \in \Sigma\right\}$ be a Cauchy net in $\left(D^{\star}(X, \mathbb{R}), \mathfrak{U}_{U C}\right)$. By Remark 3.6 the corresponding net $\left\{\sup \left(F_{\sigma}\right): \sigma \in \Sigma\right\}$ is Cauchy in $\left(Q(X, \mathbb{R}), \mathfrak{U}_{U C}\right)$. Since $\mathbb{R}$ with the Euclidean metric $d$ is complete, by [13] $\left\{\sup \left(F_{\sigma}\right): \sigma \in \Sigma\right\} \tau_{U C^{-}}$ converges to a function $f: X \rightarrow \mathbb{R}$. By Lemma 3.8 $f$ is quasicontinuous. By Proposition $3.2\left\{F_{\sigma}: \sigma \in \Sigma\right\} \tau_{U C}$-converges to $\bar{f}$. Since the space $\left(K(\mathbb{R}), H_{d}\right)$ is complete, by [13] $\left\{F_{\sigma}: \sigma \in \Sigma\right\} \tau_{U C}$-converges to a $F: X \rightarrow K(\mathbb{R})$. It is easy to verify that $F=\bar{f}$. By Theorem $2.5 F$ is densely continuous form, i.e., $\left(D^{\star}(X, \mathbb{R}), \mathfrak{U}_{U C}\right)$ is complete. By Theorem $3.7\left(\sup \left(D^{\star}(X, \mathbb{R})\right), \mathfrak{U}_{U C}\right)$ is complete.

Theorem 3.10. Let $X$ be a locally compact topological space. A subset $\mathcal{F} \subseteq$ $D^{\star}(X, \mathbb{R})$ is compact in $\left(D^{\star}(X, \mathbb{R}), \tau_{U C}\right)$ if and only if $\{\sup (F): F \in \mathcal{F}\}$ is compact in $\left(Q(X, \mathbb{R}), \tau_{U C}\right)$.

Let $\mathcal{E} \subset F(X, Y)$ and let $x \in X$, denote by $\mathcal{E}[x]$ the set $\{f(x) \in Y ; f \in \mathcal{E}\}$. We say that a subset $\mathcal{E}$ of $F(X, Y)$ is pointwise bounded [9] provided for every $x \in X, \overline{\mathcal{E}[x]}$ is compact in $(Y, d)$. 


\section{L'UBICA HOLÁ - DUŠAN HOLÝ}

If $X$ is a locally compact space and $(Y, d)$ is a metric space, the Ascoli theorem [13] says that a subset $\mathcal{E}$ of $\left(C(X, Y), \tau_{U C}\right)$ is compact if and only if it is closed in $\left(C(X, Y), \tau_{U C}\right)$, pointwise bounded and $\mathcal{E}$ is equicontinuous, where a subset $\mathcal{E}$ of $C(X, Y)$ is equicontinuous provided that for each $x \in X$ and $\epsilon>0$ there is a neighbourhood $U$ of $x$ with $d(f(x), f(z))<\epsilon$ for all $z \in U$ and $f \in \mathcal{E}$.

In [11] it is proved the Ascoli-type theorem for quasicontinuous locally bounded functions, in [9] we proved Ascoli-type theorems for quasicontinuous subcontinuous functions and in [10] we proved Ascoli-type theorems for quasicontinuous functions. To present our characterizations of compact subsets of $\left(Q(X, Y), \tau_{U C}\right)$ we need the following definition, which was introduced in [11] in the context of locally bounded functions from $F(X, Y)$.

Definition 3.11. Let $X$ be a topological space and $(Y, d)$ be a metric space. We say that a subset $\mathcal{E}$ of $F(X, Y)$ is densely equiquasicontinuous at a point $x$ of $X$ provided that for every $\epsilon>0$, there exists a finite family $\mathcal{B}$ of subsets of $X$ which are either open or nowhere dense such that $\cup \mathcal{B}$ is a neighbourhood of $x$ and such that for every $f \in \mathcal{E}$, for every $B \in \mathcal{B}$ and for every $p, q \in B$, $d(f(p), f(q))<\epsilon$. Then $\mathcal{E}$ is densely equiquasicontinuous provided that it is densely equiquasicontinuous at every point of $X$.

Remark 3.12. It is easy to prove that if $\mathcal{E}$ is a densely equiquasicontinuous subset of $F(X, Y)$, then closure of $\mathcal{E}$ with respect to the topology $\tau_{p}$ is also densely equiquasicontinuous.

We say that a system $\mathcal{E} \subset F(X, Y)$ is supported at $x \in X[10$ if for every $\epsilon>0$ there exists a neighbourhood $U(x)$ of $x$ and a finite family $\left\{\mathcal{E}_{1}, \mathcal{E}_{2}, \ldots, \mathcal{E}_{n}\right\}$ of nonempty subsets of $\mathcal{E}$ such that $\bigcup_{i=1}^{n} \mathcal{E}_{i}=\mathcal{E}$ and for every $z \in U(x)$, every $i \in\{1,2, \ldots, n\}$, and every $f, g \in \mathcal{E}_{i}, d(f(z), g(z))<\epsilon$.

Theorem 3.13 ([10]). Let $X$ be a locally compact topological space and $(Y, d)$ be a boundedly compact metric space. A subset $\mathcal{E} \subset\left(Q(X, Y), \tau_{U C}\right)$ is compact if and only if $\mathcal{E}$ is closed, pointwise bounded, there is a dense open set $M$ such that $\mathcal{E}$ is densely equiquasicontinuous at each $x \in M$ and $\mathcal{E}$ is supported at each point $x \in X \backslash M$.

Theorem 3.14 ([10]). Let $X$ be a locally compact topological space and $(Y, d)$ be a boundedly compact metric space. A subset $\mathcal{E} \subset\left(Q(X, Y), \tau_{U C}\right)$ is compact if and only if $\mathcal{E}$ is closed, pointwise bounded, there is a dense $G_{\delta}$-set $G$ such that $\mathcal{E}$ is equicontinuous at each point $x \in G$ and $\mathcal{E}$ is supported at each point $x \in X \backslash G$.

In [12] the authors proved the Ascoli-type theorem for densely continuous forms from a locally compact space to a boundedly compact metric space. In our paper we present new characterizations of compact subsets of $\left(D^{\star}(X, \mathbb{R}), \tau_{U C}\right)$ via quasicontinuous selections of elements of $D^{\star}(X, \mathbb{R})$. 


\section{QUASICONTINUOUS FUNCTIONS, DENSELY CONTINUOUS FORMS AND...}

TheOREM 3.15. Let $X$ be a locally compact topological space. A subset $\mathcal{F} \subseteq\left(D^{\star}(X, \mathbb{R}), \tau_{U C}\right)$ is compact if and only if $\{\sup (F): F \in \mathcal{F}\}$ is closed in $\left(Q(X, \mathbb{R}), \tau_{U C}\right)$, pointwise bounded, there is a dense open set $M$ such that $\{\sup (F): F \in \mathcal{F}\}$ is densely equiquasicontinuous at each $x \in M$ and is supported at each point $x \in X \backslash M$.

TheOrem 3.16. Let $X$ be a locally compact topological space. A subset $\mathcal{F} \subseteq$ $\left(D^{\star}(X, \mathbb{R}), \tau_{U C}\right)$ is compact if and only if $\{\sup (F): F \in \mathcal{F}\}$ is closed in $\left(Q(X, \mathbb{R}), \tau_{U C}\right)$, pointwise bounded, there is a dense $G_{\delta}$-set $G$ such that $\{\sup (F): F \in \mathcal{F}\}$ is equicontinuous at each point $x \in G$ and is supported at each point $x \in X \backslash G$.

\section{REFERENCES}

[1] BEER, G.: Topologies on Closed and Closed Convex Sets. Kluwer Acad. Publ., 1993.

[2] BORSÍK, J.: Points of continuity, quasicontinuity and cliquishness, Rend. Istit. Math. Univ. Trieste 26 (1994), 5-20.

[3] BOUZIAD, A.: Every Čech-analytic Baire semitopological group is a topological group, Proc. Amer. Math. Soc. 124 (1996), 953-959.

[4] CRANnelL, A.-FRANTZ, M.-LEMASURIER, M.: Closed relations and equivalence classes of quasicontinuous functions, Real Anal. Exchange 31 (2006/2007), 409-424.

[5] GILES, J. R.-BARTLETT, M. O.: Modified continuity and a generalization of Michael's selection theorem, Set-Valued Anal. 1 (1993), 247-268.

[6] HOLÁ, L'.: Functional characterization of p-spaces, Cent. Eur. J. Math. 11 (2013), 2197-2202.

[7] HOLÁ, L.-HOLÝ, D.: Minimal USCO maps, densely continuous forms and upper semicontinuous functions, Rocky Mount. Math. J. 39 (2009), 545-562.

[8] HOLÁ, L'--HOLÝ, D.: New characterization of minimal CUSCO maps, Rocky Mount. Math. J. 44 (2014), 1851-1866.

[9] HOLÁ, L'-HOLÝ, D.: Quasicontinuous subcontinuous functions and compactness, Mediterranean J. Math. 13 (2016), 4509-4518.

[10] HOLÁ, L.-HOLÝ, D.: Quasicontinuous functions and compactness (submitted).

[11] HOLÝ, D.: Ascoli-type theorems for locally bounded quasicontinuous functions, minimal usco and minimal cusco maps, Ann. Funct. Anal. 6 (2015), 29-41, http://projecteuclid.org/afa

[12] HAMMER, S. T.-MCCOY, R. A.: Spaces of densely continuous forms, Set-Valued Anal. 5 (1997), 247-266.

[13] KELlEY, J. L.: General Topology. Van Nostrand, New York, 1955.

[14] KEMPISTY, S.: Sur les fonctions quasi-continues, Fund. Math. 19 (1932), 184-197.

[15] MATEJDES, M.: Minimality of multifunctions, Real Anal. Exchange 32 (2007), $519-526$. 


\section{L'UBICA HOLÁ - DUŠAN HOLÝ}

[16] MOORS, W. B.: Any semitopological group that is homeomorphic to a product of Čech-complete spaces is a topological group, Set-Valued Var. Anal. 21 (2013), 627-633.

[17] MOORS, W. B.: Fragmentable mappings and CHART groups, Fund. Math. 234 (2016), 191-200.

[18] MOORS, W. B.: Semitopological groups, Bouziad spaces and topological groups, Topology Appl. 160 (2013), 2038-2048.

[19] NEUBRUNN, T.: Quasi-continuity, Real Anal. Exchange 14 (1988), 259-306.

[20] VAUGHAN, H.: On locally compact metrizable spaces, Bull. Amer. Math. Soc. 43 (1937), 532-535.

Received December 12, 2016

L'ubica Holá

Academy of Sciences

Institute of Mathematics

Štefánikova 49

SK-814-73 Bratislava

SLOVAKIA

E-mail: hola@mat.savba.sk

Dušan Holý

Department of Mathematics and

Computer Science

Faculty of Education

Trnava University

Priemyselná 4

SK-918-43 Trnava

SLOVAKIA

E-mail: dusan.holy@truni.sk 